\title{
ÉTUDE DE LA GÉNÉRATION D'HARMONIQUES EN ONDE DE SURFACE DANS DES CRISTAUX PIÉZOÉLECTRIQUES
}

\author{
G. COUSSOT \\ Laboratoire Central de Recherches THOMSON-CSF. Domaine de Corbeville, 91401 Orsay, France \\ E. BRIDOUX
}

Centre Universitaire de Valenciennes, 59300 Valenciennes, France

\begin{abstract}
Résumé. - Par analogie avec les ondes acoustiques se propageant dans un liquide, une analyse unidimensionnelle des phénomènes non linéaires en onde de Rayleigh est présentée. Dans le modèle proposé, un seul paramètre caractérise la non-linéarité du matériau étudié. La valeur de ce paramètre, une fois connue, permet d'interpréter l'ensemble des résultats expérimentaux obtenus sur $\mathrm{LiNbO}_{3}$ et $\mathrm{Bi}_{12} \mathrm{GeO}_{20}$ : générations d'harmoniques, phénomènes de saturation dus à l'amplitude finie des ondes acoustiques.
\end{abstract}

Abstract. - Using a theoretical technique developed for liquids, one one-dimensional model containing one non-linear parameter is proposed for harmonic generation of acoustic surface waves. A value of the single parameter is found by $\mathrm{LiNbO}_{3}, \mathrm{Bi}_{12} \mathrm{GeO}_{20}$ which gives good agreement between the model predictions and the experimental results : generation and limitation of harmonics due to the finite-amplitude effects.

L'excitation des ondes élastiques de Rayleigh par de simples électrodes métalliques interdigitées, déposées sur un cristal piézoélectrique, a considérablement élargi le champ d'étude et d'application des phénomènes acoustiques depuis quelques années. Un intérêt particulièrement important est l'utilisation de ces ondes pour réaliser des systèmes électroniques grâce à leur faible vitesse de propagation, à leur amplification et à la possibilité de guidage. Il est donc nécessaire de bien connaître les caractéristiques de propagation de ces ondes de surface. En particulier, dans un cristal réel, les équations correspondantes du mouvement ne sont plus linéaires et n'admettent aucune solution harmonique simple à partir d'un certain seuil de puissance acoustique. Une conséquence importante est la création d'ondes harmoniques associées à la propagation de l'onde fondamentale.

Ce travail rapporte une étude théorique et des mesures d'effets anharmoniques (ou non linéaires) pour différents substrats, obtenues à l'aide d'une sonde optique.

Dans la première partie, nous développerons une approche théorique en proposant un modèle unidimensionnel ne faisant intervenir qu'un seul paramètre caractérisant la non-linéarité du matériau. Ce modèle sera testé pour différents matériaux au cours de l'étude expérimentale présentée dans la seconde partie.
1. Approche phénoménologique des phénomènes non linéaires. - Le calcul rigoureux des phénomènes non Jinéaires au cours de la propagation des ondes élastiques nécessite la résolution de l'équation du déplacement de Thurston [1] pour une onde d'amplitude finie. L'application de ce formalisme aux ondes élastiques de Rayleigh est difficile à cause de la structure complexe de ce type d'onde. En outre, l'utilisation de substrats piézoélectriques rend le problème inextricable. Aussi avons-nous considéré un modèle phénoménologique unidimensionnel en introduisant une constante de non-linéarité caractéristique du matériau sans chercher toutefois à la relier aux constantes élastiques $\mathrm{du}$ second ordre et du troisième ordre.

1.1. GÉNÉRATION DE L'HARMONIQUe D'ORDRE 2. Nous allons tout d'abord envisager un modèle, très simple, dans lequel nous supposons que l'atténuation linéaire et la dispersion sont négligeables, et que seule l'harmonique d'ordre 2 croît. Nous admettons qu'un modèle scalaire est une représentation convenable et nous choisissons comme variable la composante de la déformation $S$ normale à la surface libre. Si $v_{s}$ est la vitesse de phase de l'onde élastique, nous avons :

$$
\frac{\partial^{2} S}{\partial t^{2}}=v_{\mathrm{s}}^{2} \frac{\partial^{2} S}{\partial z^{2}}
$$


Le fait que $S$ décroît avec la profondeur de pénétration d'une façon complexe est implicitement contenu dans $v_{\mathrm{s}}$. En introduisant un terme quadratique dans l'équation de propagation (1), nous obtenons :

$$
\frac{\partial^{2} S}{\partial t^{2}}=v_{\mathrm{s}}^{2} \frac{\partial^{2}\left[S+\beta S^{2}\right]}{\partial z^{2}}
$$

où $\beta$ est un paramètre non linéaire qui doit être déterminé expérimentalement. Celui-ci représente physiquement une mesure du rapport entre les constantes élastiques du troisième ordre et les constantes élastiques durcies. Ecrivons que la déformation totale est une combinaison des déformations de l'onde fondamentale de pulsation $\omega_{1}$ et de la $2^{\mathrm{e}}$ harmonique de pulsation $\omega_{2}$ se propageant suivant $z$ :

$$
\begin{array}{r}
S=X_{1} \cos \left[k_{1} z-\omega_{1} t\right]+Y_{1} \sin \left[k_{1} z-\omega_{1} t\right]+ \\
+X_{2} \cos \left[k_{2} z-\omega_{2} t\right]+Y_{2} \sin \left[k_{2} z-\omega_{2} t\right] .
\end{array}
$$

En reportant (3) dans (2) et en remarquant que seul $X_{1} \neq 0$ à l'origine, la résolution de ce système conduit à deux équations :

$$
\frac{\partial}{\partial z}\left[\begin{array}{c}
X_{1} \\
Y_{2}
\end{array}\right]=\frac{1}{2} \beta k_{1}\left[\begin{array}{cc}
-X_{1} & Y_{2} \\
X_{1}^{2} &
\end{array}\right] \quad k_{1}=\frac{\omega_{1}}{v_{s}}
$$

Cette forme réduite est facilement exploitable : le paramètre non linéaire peut être calculé par approximation à partir des mesures expérimentales des déformations, l'amplitude de la déformation initiale étant obtenue à partir de la puissance acoustique fournie et des pertes des transducteurs.

1.2. GÉnÉration DE PLUSIEURS harMoniQues. Lorsque la puissance acoustique augmente, il faut tenir compte de la génération des harmoniques d'ordre supérieur de sorte que la déformation résultante s'écrit :

$$
\begin{aligned}
S=\sum S_{i}=\sum_{i} X_{i}(z) \cos \left[k_{i} z-\omega_{i} t\right]+ \\
+Y_{i}(z) \sin \left[k_{i} z-\omega_{i} t\right]
\end{aligned}
$$

où les amplitudes des dilatations $X_{i}, Y_{i}$ varient lentement de façon que :

$$
X_{i} \gg \frac{1}{k_{i}} \frac{\partial X_{i}}{\partial z} \gg \frac{1}{k_{i}^{2}} \frac{\partial^{2} X_{i}}{\partial z^{2}} \ldots
$$

Posons

$$
\begin{aligned}
& \mathrm{Co}(i)=\cos \left[k_{i} z-\omega_{i} t\right] \\
& \mathrm{Si}(i)=\sin \left[k_{i} z-\omega_{i} t\right]
\end{aligned}
$$

de sorte que :

$$
S=\sum_{i} X_{i} \operatorname{Co}(i)+Y_{i} \operatorname{Si}(i)
$$

Après avoir calculé $S^{2}$, nous avons :

$$
\frac{\partial^{2} S}{\partial t^{2}}=\sum_{i}-\omega_{i}^{2} S_{i}
$$

$$
\begin{aligned}
\frac{\partial^{2} S}{\partial z^{2}}=\sum_{i}\left[-k_{i}^{2}\right. & \left.X_{i}+2 k_{i}\left(\frac{\partial Y_{i}}{\partial z}\right)\right] \operatorname{Co}(i) \times \\
\times & {\left[-k_{i}^{2} Y_{i}-2 k_{i}\left(\frac{\partial X_{i}}{\partial z}\right)\right] \operatorname{Si}(i) }
\end{aligned}
$$

et

$$
\begin{aligned}
\frac{\partial^{2}\left(S^{2}\right)}{\partial z^{2}} & = \\
=\sum_{i} \sum_{j} & {\left[-\frac{1}{2}\left(k_{i}+k_{j}\right)^{2}\left(X_{i} X_{j}-Y_{i} Y_{j}\right) \operatorname{Co}(i+j)\right.} \\
& -\frac{1}{2}\left(k_{i}-k_{j}\right)^{2}\left(X_{i} X_{j}+Y_{i} Y_{j}\right) \mathrm{Co}(i-j) \\
& -\frac{1}{2}\left(k_{i}+k_{j}\right)^{2}\left(X_{i} Y_{j}+Y_{i} X_{j}\right) \mathrm{Si}(i+j) \\
& \left.-\frac{1}{2}\left(k_{i}-k_{j}\right)^{2}\left(Y_{i} X_{j}-X_{i} Y_{j}\right) \mathrm{Si}(i-j)\right] .
\end{aligned}
$$

Dans (10) et (11), nous avons négligé les termes dérivés en accord avec (6). Le développement du terme $\partial^{2}\left(S^{2}\right) / \partial z^{2}$ met bien en évidence l'effet de mélange dû à la non-linéarité qui produit des ondes de fréquences somme et différence. Remarquons qu'il n'y a pas de dispersion dans le domaine de fréquences considéré (longueur d'onde très inférieure à la distance atomique, défauts de surface négligeables) de sorte que :

$$
\omega_{i}^{2}-k_{i}^{2} v_{s}^{2}=0 .
$$

Pour tenir compte des pertes dans le milieu, introduisons un coefficient d'atténuation $\alpha_{i}$ et nous obtenons après substitution dans l'éq. (2), les relations suivantes :

$$
\begin{aligned}
& \frac{\partial X_{i}}{\partial z}=-\alpha_{i} X_{i}+\frac{\beta}{2 k_{i}}\left[\frac{\partial^{2}\left(S^{2}\right)}{\partial z^{2}}\right]_{\mathrm{Si}(i)} \\
& \frac{\partial Y_{i}}{\partial z}=-\alpha_{i} Y_{i} \mp \frac{\beta}{2 k_{i}}\left[\frac{\partial^{2}\left(S^{2}\right)}{\partial z^{2}}\right]_{\mathrm{Co}(i)}
\end{aligned}
$$

où les suffixes $\mathrm{Si}(i)$ et $\mathrm{Co}(i)$ représentent les tenues en $\mathrm{Si}(i)$ et $\mathrm{Co}(i)$ de l'éq. (11).

Supposons que l'onde fondamentale varie suivant une loi en cosinus, les harmoniques impaires varieront suivant une loi en cosinus et les harmoniques paires en sinus. En se limitant à la quatrième harmonique et en désignant par $S_{1} \ldots S_{4}$ les déformations associées, (5) s'écrit :

$\frac{\mathrm{d}}{\mathrm{d} z}\left[\begin{array}{l}\mathrm{S}_{1} \\ \mathrm{~S}_{2} \\ \mathrm{~S}_{3} \\ \mathrm{~S}_{4}\end{array}\right]=\left[\begin{array}{l}-\alpha_{1} \mathrm{~S}_{1}-\frac{1}{2} \beta k_{1}\left(\mathrm{~S}_{2} \mathrm{~S}_{1}-\mathrm{S}_{3} \mathrm{~S}_{2}+\mathrm{S}_{4} \mathrm{~S}_{3}\right) \\ -\alpha_{2} \mathrm{~S}_{2}+\frac{1}{2} \beta k_{1}\left(\mathrm{~S}_{1}^{2}+2 \mathrm{~S}_{3} \mathrm{~S}_{1}+2 \mathrm{~S}_{4} \mathrm{~S}_{2}\right) \\ -\alpha_{3} \mathrm{~S}_{3}-\frac{1}{2} \beta k_{1}\left(3 \mathrm{~S}_{4} \mathrm{~S}_{1}+3 \mathrm{~S}_{2} \mathrm{~S}_{1}\right) \\ -\alpha_{4} \mathrm{~S}_{4}+\frac{1}{2} \beta k_{1}\left(-2 \mathrm{~S}_{2}^{2}+4 \mathrm{~S}_{1} \mathrm{~S}_{3}\right)\end{array}\right]$ 
Si les conditions expérimentales initiales sont connues (puissance acoustique, fréquence, atténuation), nous devons trouver une seule valeur de $\beta$ satisfaisant (15).

1.3. CAS GÉNÉRAL. - La méthode précédente n'est valable que si la puissance acoustique fondamentale est suffisamment faible $(<1 \mathrm{~W})$. Pour des puissances plus élevées, des phénomènes de saturation apparaissent et ne peuvent être expliquées par les modèles proposés. Par analogie, avec l'étude de la propagation d'onde d'amplitude finie dans les fluides visqueux, écrivons l'équation du mouvement à une dimension :

$$
\frac{\partial^{2} u}{\partial t^{2}}=v_{\mathrm{s}}^{2} \frac{\partial^{2} u}{\partial z^{2}}+v_{\mathrm{s}}^{2} \beta \frac{\partial u}{\partial z} \frac{\partial^{2} u}{\partial z^{2}}+\frac{2 \alpha v_{\mathrm{s}}^{3}}{\omega^{2}} \frac{\partial^{3} u}{\partial z^{2} \partial t}
$$

où $\beta$ est le paramètre caractéristique de la non-linéarité du matériau, $\alpha$ l'atténuation de l'onde acoustique à la pulsation $\omega$. En admettant que $\alpha / \omega^{n}$ est constant, l'éq. (16) peut être ramenée à l'équation de Burger dont on connaît la solution exacte. Lorsque l'onde acoustique est initialement sinusoïdale, la méthode consiste à déterminer les composantes de Fourier de la déformation se propageant dans le cristal. Blackstock [2] a ainsi développé une solution numérique. Cette méthode a été reprise par Wilkinson [3] et appliquée avec succès à la génération des harmoniques d'ondes longitudinales de volume. Adaptons ce modèle aux ondes de Rayleigh. Pour cela, nous définissons une longueur de discontinuité :

$$
L_{0}=\frac{2 v_{\mathrm{s}}^{2}}{\beta \omega_{1} V_{1}(0)}
$$

où $V_{1}(0)$ est obtenu à partir de la relation suivante :

$$
\frac{\partial u}{\partial t}=\sum_{i} V_{i}(z) \sin \left(k_{i} z-\omega_{i} t\right) .
$$

En reportant (18) dans (16) on obtient une équation de type Burger que Blackstock a résolue en calculant les $V_{n}$ par analyse harmonique de la fonction $V$. Nous avons transcrit en Fortran IV pour IBM 1130 [4] le programme de Blackstock qui nous permet de calculer $V_{n}$ en fonction de la distance réduite parcourue $\sigma=z / L_{0}$ et du paramètre $\beta$. Nous obtenons ainsi la variation des amplitudes des seconde et troisième harmoniques

$$
B_{2}=\frac{V_{2}(\sigma)}{V_{1}(0)}, \quad B_{3}=\frac{V_{3}(\sigma)}{V_{1}(0)}
$$

pour une onde d'amplitude initiale $V_{1}(0)$. Ces variations normalisées sont reportées sur la figure 1 pour des valeurs de $\beta$ variant de 0,5 à 0,8 .

La comparaison des résultats expérimentaux et des courbes théoriques fournissent une valeur de $\beta$.

On peut montrer que lorsque $\sigma \ll 1$ :

$$
\frac{V_{2}(z)}{V_{1}(0)}=\frac{1}{\alpha_{1} L_{0}}\left(\mathrm{e}^{-2 \alpha_{1} z}-\mathrm{e}^{-4 \alpha_{1} z}\right)
$$

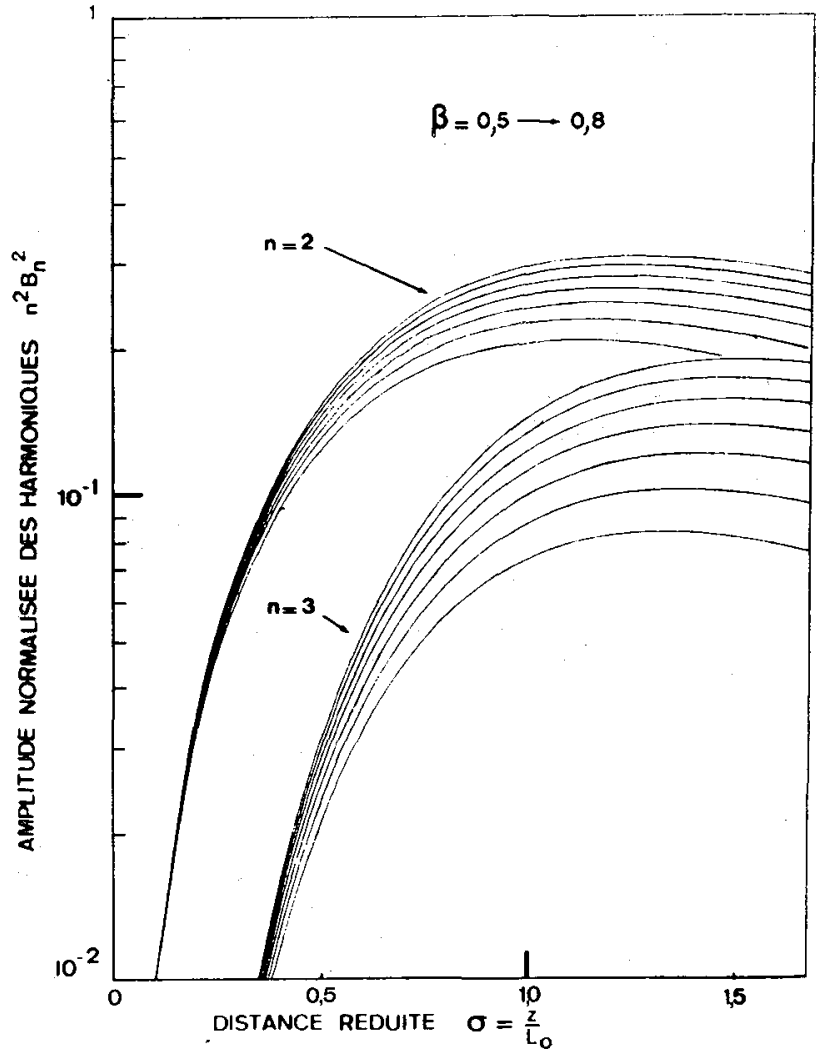

FIG. 1. - Amplitude normalisée des seconde et troisième harmoniques d'une onde élastique fondamentale d'amplitude finie en fonction de la distance réduite $\sigma$ pour $\beta$ variant de 0,5 à 0,8 par pas de 0,05 augmentant vers le haut de la figure.

si l'atténuation $\alpha_{1}$ d'une onde de petite amplitude est faible :

$$
\frac{V_{2}(z)}{V_{1}(0)}=\frac{1}{2} \frac{\mathrm{S}_{2}(z)}{\mathrm{S}_{1}(0)}=\frac{1}{4} \beta k \mathrm{~S}_{1}(0) z
$$

équation identique à l'éq. (4).

2. Etude expérimentale. - Pour mettre en évidence la croissance et l'évolution des harmoniques d'une onde de Rayleigh, nous avons utilisé une sonde optique. Le principe de cette technique est fondé sur la diffraction d'un faisceau lumineux (laser) par la déformation de la surface et par la variation d'indice lors de la propagation des ondes sur le substrat [5]. Cette déformation est assimilée à un réseau optique et la relation angulaire entre la lumière incidente et la lumière diffractée s'écrit simplement :

$$
\sin \theta_{m}=\sin \theta_{0}+m \frac{\lambda}{\Lambda} \quad m=1,2, \ldots
$$

où $\theta_{m}$ l'angle de diffraction à l'ordre $m, \theta_{0}$ l'angle d'incidence, $\lambda$ et $A$ les longueurs d'onde optique et acoustique. L'intensité de la lumière diffractée par réflexion à l'ordre $m$ est alors :

$$
\frac{I_{m}}{I_{0}}=\sum_{n, l} \frac{1}{(l !)^{2}}\left[\frac{A}{\left(n f_{1}\right)^{2}} \cdot P_{\mathrm{d}}\left(n f_{1}\right)\right]^{l}
$$


où la sommation porte sur tous les entiers $l$ et $n$, tels que $l \times n=m, A$ est un facteur de proportionnalité dépendant des constantes optiques et acoustiques du cristal, $P_{\mathrm{d}}$ la densité de puissance acoustique de l'harmonique $n, f_{1}$ la fréquence acoustique fondamentale et $I_{0}$ l'intensité lumineuse incidente.

Il faut remarquer que l'intensité de la lumière diffractée aux ordres supérieurs à 1 , a deux origines : l'une est due à la diffraction de la lumière par le signal acoustique à la fréquence fondamentale $f_{1}$ donnée par l'éq. $\left(20^{\prime}\right)$ pour $m>1$, l'autre à la diffraction de la lumière par les harmoniques de fréquences $n f_{1}$ pour $m=1$. Cette seconde contribution est prépondérante, dans un rapport 100 environ, pour des puissances acoustiques de l'ordre de $1 \mathrm{~W}$.

Cette remarque nous autorise ainsi à étudier la génération d'ondes harmoniques dans le cristal en détectant, aux angles correspondants $n \theta_{1}$, la lumière diffractée par l'harmonique dont l'intensité est :

$$
\frac{I_{n}}{I_{0}}=\frac{A}{\left(n f_{1}\right)^{2}} P_{\mathrm{d}}\left(n f_{1}\right)
$$

A partir du vecteur de Poynting défini dans la direction $z$, nous obtenons la puissance acoustique par unité de largeur du transducteur.

D'autre part, il est possible d'exprimer la compo-

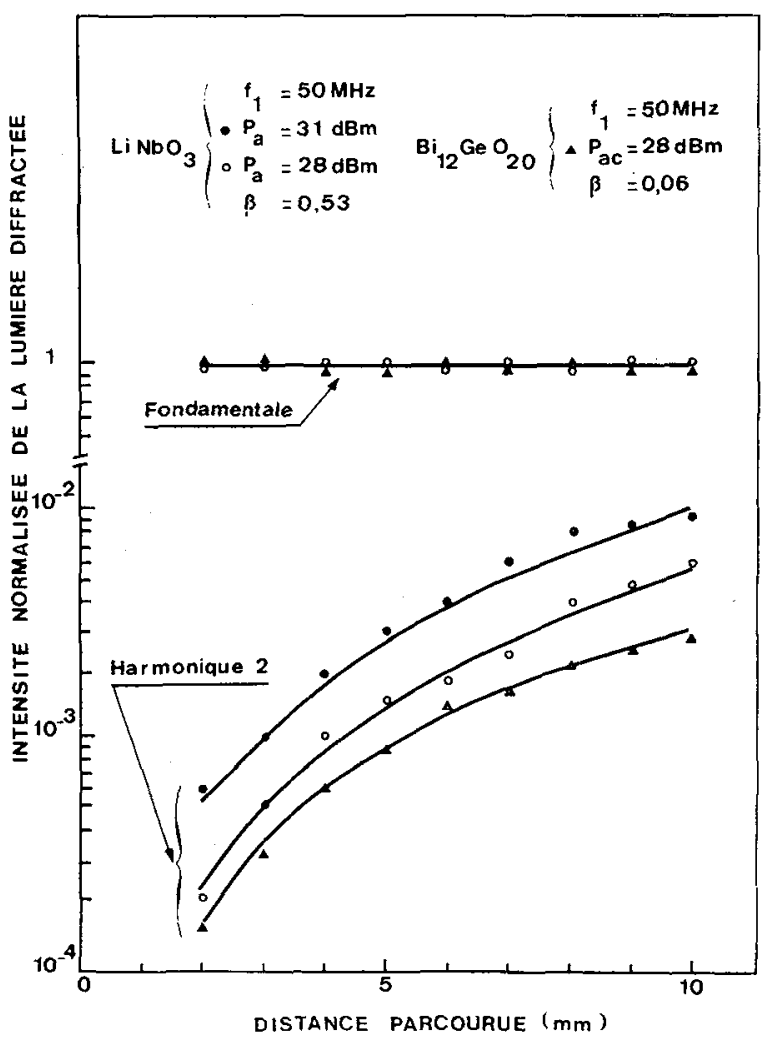

FIG. 2. - Génération de la seconde harmonique dans $\mathrm{Bi}_{12} \mathrm{GeO}_{20}$ et $\mathrm{LiNbO}_{3}$ pour de faibles puissances acoustiques. Les courbes tracées sont obtenues à partir du modèle théorique simple.

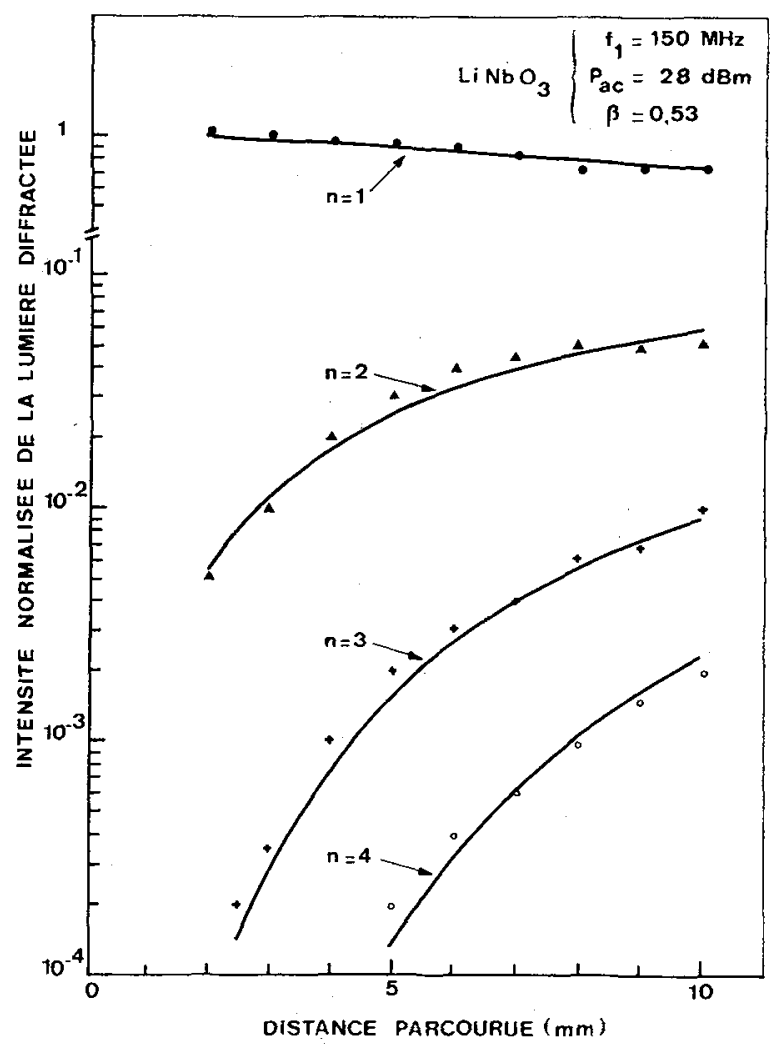

FIG. 3. - Génération de plusieurs harmoniques dans $\mathrm{LiNbO}_{3}$. Les courbes en trait plein correspondent aux prédictions théoriques.

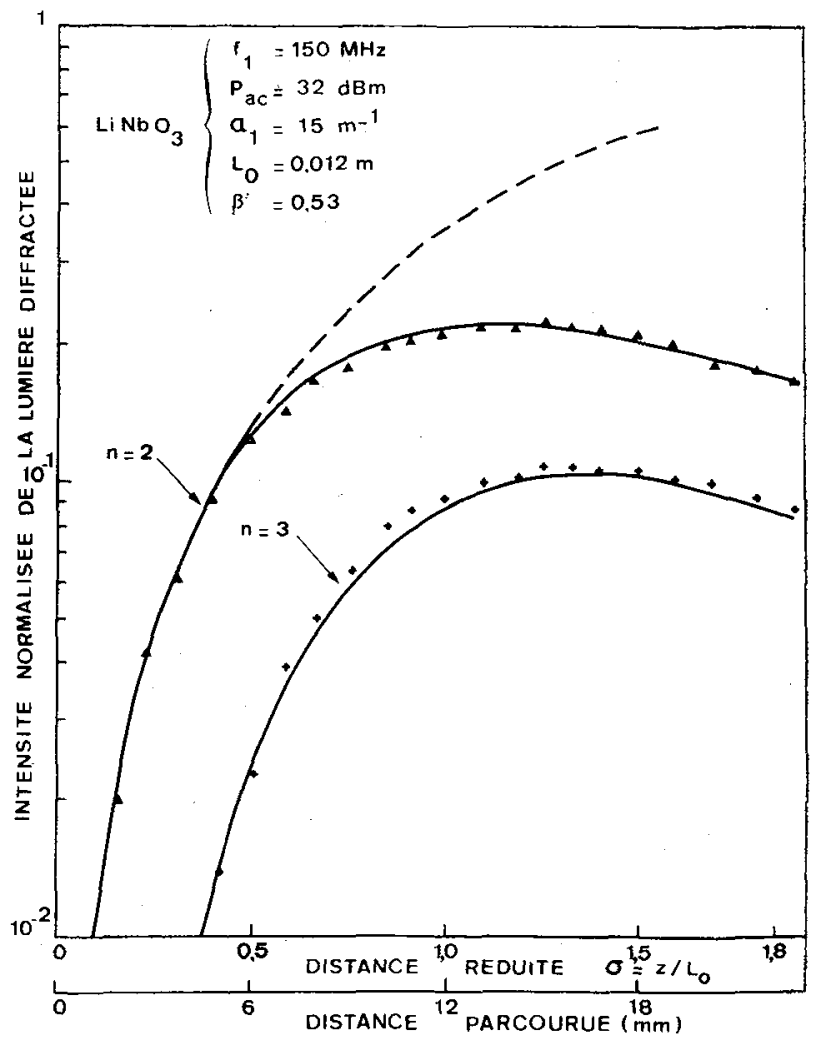

Fig. 4. - Génération des seconde et troisième harmoniques dans $\mathrm{LiNbO}_{3}$ pour une puissance acoustique élevée. Le phénomène de saturation est bien résolu et les courbes théoriques déduites de la figure 1 sont dessinées en trait plein. La courbe en pointillés correspond au modèle théorique simple. 
sante normale du déplacement mécanique en fonction de la densité de puissance acoustique :

$$
u^{2}=K \frac{P_{\mathrm{ac}}}{\omega \cdot d}
$$

$d$ étant la largeur active des transducteurs égale à $2 \mathrm{~mm}$. La constante $K$ dépend du matériau et de la direction cristallographique. Pour une coupe $Y$ et une propagation suivant $Z$ d'un cristal de $\mathrm{LiNbO}_{3}$, $K=1,26 \times 10^{-11}$; pour une coupe [1]10] et une propagation suivant [001] d'un cristal de $\mathrm{Bi}_{12} \mathrm{GeO}_{20}$, $K=2,55 \times 10^{-11}$.

Le rapport des intensités lumineuses devient:

$$
\frac{I_{n}(z)}{I_{1}(0)}=\left[\frac{u_{n}(z)}{u_{1}(0)}\right]^{2}
$$

$u_{1}$ étant la valeur initiale de la composante normale du déplacement de l'onde fondamentale. L'intensité de la lumière diffractée normalisée $n^{2} I_{n}(z) / I_{1}(0)$ est mesurée en fonction de la distance parcourue par l'onde élastique sur des échantillons de $\mathrm{LiNbO}_{3}$ et de $\mathrm{Bi}_{12} \mathrm{GeO}_{20}$ pour plusieurs fréquences fondamentales et différents niveaux de puissance. Pour des fréquences de $50 \mathrm{MHz}$ et des puissances faibles $(<1 \mathrm{~W})$, les résultats détaillés ont déjà été publiés [6], [7]. Seule la seconde harmonique est détectée et l'atténuation est négligeable (Fig. 2). L'accord entre les résultats théoriques et expérimentaux est obtenu pour $\beta=0,06\left(\mathrm{Bi}_{12} \mathrm{GeO}_{20}\right)$ et $\beta=0,53$ $\left(\mathrm{LiNbO}_{3}\right)$. A $150 \mathrm{MHz}$, pour une puissance acoustique de $28 \mathrm{dBm}$ sur un nouvel échantillon de $\mathrm{LiNbO}_{3}$, des mesures précises jusqu'au troisième harmonique ont montré un excellent accord entre les prédictions théoriques et expérimentales en tenant compte de l'atténuation toujours pour la même valeur de $\beta$ (Fig. 3).

Cependant, une augmentation de la puissance acoustique fait apparaître un phénomène de saturation dans la croissance des harmoniques (Fig. 4), l'application $\mathrm{du}$ modèle théorique général à ces résultats nous conduit encore à une valeur de $\beta=0,53$ pour $\mathrm{LiNbO}_{3}$. Ainsi le paramètre $\beta$ reflète bien la non-linéarité du cristal étudié quelles que soient la fréquence, l'atténuation de l'onde acoustique fondamentale à faible niveau, la puissance acoustique incidente. Sa valeur obtenue par comparaison des résultats expérimentaux et théoriques est un critère de la non-linéarité du matériau considéré.

Remerciements. - Nous remercions le Dr Wilkinson qui a eu l'amabilité de nous transmettre un exemplaire de thèse.

\section{Bibliographie}

[1] Thurston R. N., dans Physical Acoustics, Warren P. Mason Ed. (Academic Press Inc, New York 1964), vol. I-A, chap. 1, 1-110.

[2] Blackstock D. T., J. Acoust. Soc. Am. 36 (1966) 634.

[3] Wilkinson C. D. W., Thèse de Doctorat, Université de Stanford (1968).

[4] Bridoux E., Thèse de doctorat, Université de Lille (1970).
[5] Slobodnik A. J., J. Acoust. Soc. Am. 48 (1969) 204. Lean E. G. H. and Tseng C. C., Proc. of IEEE 58 (1970) 1939.

[6] Bridoux E., Rouvaen J. M., Coussot G. et DieuLESAINT E., Appl. Phys. Lett. 19 (1971) 523.

[7] Adler E., Bridoux E., Coussot G. et Dieulesaint E., à paraître dans IEEE Trans. Sonics and Ultrasonics. 